\title{
Operational aspects of the VELO cooling system of LHCb.
}

\section{Eddy Jans ${ }^{1}$}

Nikhef, Science Park 105, 1098 XG Amsterdam, The Netherlands

E-mail: e.jansenikhef.nI

The VELO is a silicon strip detector that is positioned around the interaction region of LHCb. It is placed inside a secondary vacuum with respect to that of the LHC. The cooling system of the VELO is based on the bi-phase accumulator controlled method, using $\mathrm{CO}_{2}$ as coolant. The main objective is the removal of the heat produced by the front-end electronics. Moreover, the leakage currents of the sensors are strongly reduced and thermal runaway is prevented. Since the sensors have been irradiated in Run 1 they should always be cooled to below $-5{ }^{\circ} \mathrm{C}$. The operational principle and main characteristics of the system are described, as well as the warning and safety systems that guarantee the safe operation of the detector. The few problems that have been encountered during the four years of continuous operation are discussed together with the solutions that have been implemented.

22nd International Workshop on Vertex Detectors, September 15-20, 2013

Lake Starnberg, Germany

\footnotetext{
${ }^{1}$ on behalf of the LHCb VELO group.
} 


\section{Introduction}

The vertex locator (VELO) of LHCb is the detector [1] that envelops the interaction region of the Large Hadron Collider (LHC) at Point 8. Its main task is to determine the positions of the primary vertices from the initial $p p$ collisions and of the secondary vertices from the decays of beauty and charm particles. It consists of two movable detector halves, each equipped with 21 modules. Every module contains two half-circular silicon sensors with 2048 strips. In the upstream region there are 4 additional single-sided modules of the Pile-Up detector. Each of the 88 sensors is read out by 16 Beetle front-end chips [2]. The heat produced by a double-sided VELO module amounts to $\sim 20 \mathrm{~W}$. On each module the temperature is measured at four positions. The core of the substrate of a module is made of $400 \mu \mathrm{m}$ thick thermal pyrolytic graphite (TPG). It carries the heat load from the front-end chips to the five aluminum cooling blocks that encase stainless steel cooling capillaries with an inner and outer diameter of 1.0 and $1.5 \mathrm{~mm}$, respectively (see Fig. 1).

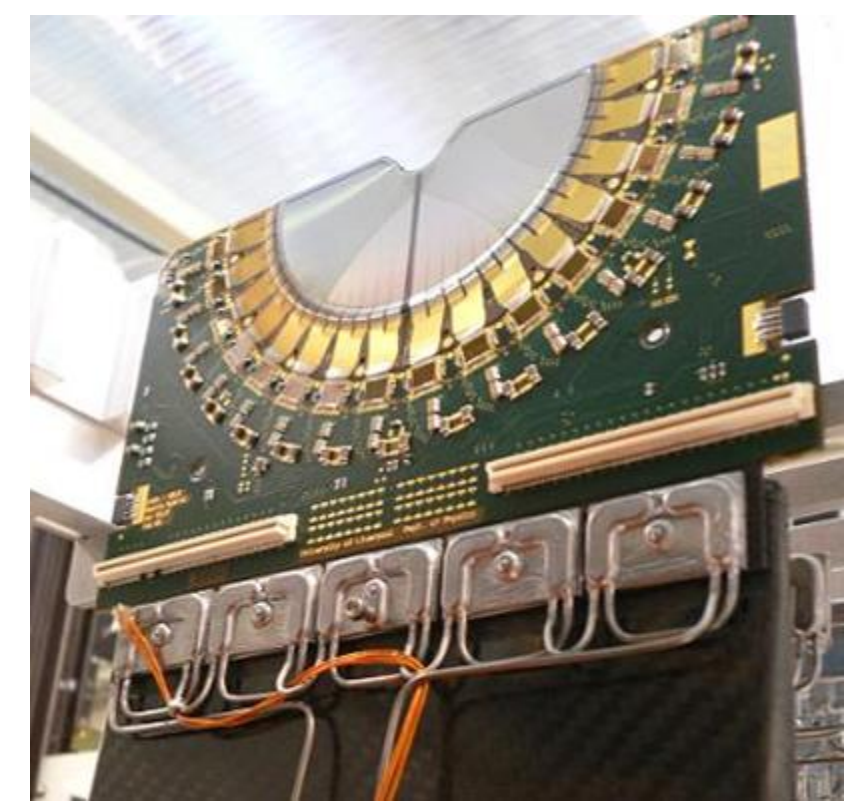

Fig. 1: VELO module with a half-circular silicon sensor, 16 Beetle readout chips, five cooling blocks and the capillaries for the $\mathrm{CO}_{2}$ supply.

In the cooling blocks evaporative $\mathrm{CO}_{2}$ cooling provides sufficient cooling power to keep the sensors under operational conditions at $-7{ }^{\circ} \mathrm{C}$. The detector is operated in a secondary vacuum of $\sim 3 \times 10^{-7}$ mbar.

In section 2 a short description of the most important aspects of the cooling system is given. Some key figures of the performance are presented in section 3. The importance of filters throughout the system is demonstrated in section 4. Section 5 describes the phenomenon of super-heated $\mathrm{CO}_{2}$. The way in which the implementation of redundant components has increased the reliability of the cooling system is discussed in section 6 . Due to radiation damage the sensors should continuously be kept below $-5{ }^{\circ} \mathrm{C}$, a topic that is briefly addressed in section 7. Some details of the warning and safety systems are presented in section 8 . Finally, in section 9, the conclusions and outlook are presented. 


\section{Cooling system}

The modules are cooled by a cooling system operating with bi-phase $\mathrm{CO}_{2}$. The advantages of $\mathrm{CO}_{2}$ as cooling agent are its extremely good radiation hardness and the small amount of material needed to supply the required latent heat. A schematic diagram of the system is shown in Fig. 2.

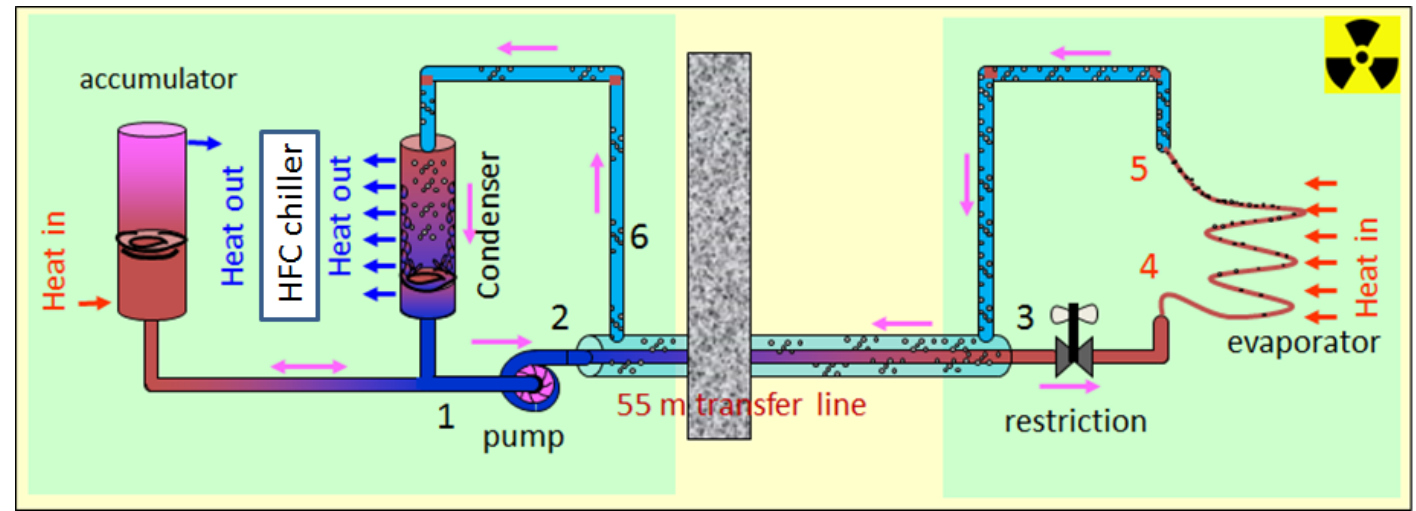

Fig. 2: Layout of VELO cooling system: cooling plant on the left, shielding wall and transfer line in the middle, and the evaporator in the detector on the right.

The basic cooling loop consists of an accumulator that always contains a saturated mixture of vapor and liquid, a pump, a restriction, an evaporator and a condenser. Sub-cooled ${ }^{2}$ $\mathrm{CO}_{2}$ is mechanically pumped from the cooling plant via a $55 \mathrm{~m}$ long concentric transfer line to the detector, where it passes through a restriction and is brought to boiling. The boiling temperature is determined by the pressure in the evaporator, which is almost identical to the pressure in the accumulator. The 23 capillaries of one detector side are connected in parallel. The $\mathrm{CO}_{2}$ flows through the capillaries in the cooling blocks and removes the heat. The nominal operational temperature is $-28{ }^{\circ} \mathrm{C}$ and the mass flow per module is $0.43 \mathrm{~g} / \mathrm{s}$, which corresponds to $130 \mathrm{~W}$ in the case of full evaporation. The outlets of the cooling tubes are combined and the bi-phase mixture returns via the outer concentric tube of the transfer line back to the condenser in the cooling plant, where the enthalpy is reduced by freon (R507A) of about $-42{ }^{\circ} \mathrm{C}$, supplied by a water-cooled chiller.

Thanks to the low hydraulic resistance between the evaporator outlet and the accumulator, the pressure, and therefore the temperature, in the evaporator is close to that in the accumulator. The corresponding offset amounts to 2 degrees. A heat load of $400 \mathrm{~W}$ can be cooled at $-30{ }^{\circ} \mathrm{C}$ per detector side.

All cold tubes that are in contact with air have been thermally insulated. The 55 meter long transfer lines are encapsulated by foamglass, which is subsequently covered by an aluminum shield for protection. Originally the tubes in the freon and $\mathrm{CO}_{2}$ plants, as well as the $\mathrm{CO}_{2}$ pipes at the detector side were covered by Armaflex ${ }^{3} \mathrm{NH}$, as this was imposed by the CERN safety regulations. However, this materiel dried out quickly and became an open-cell structure. After obtaining special permission, this insulation was replaced by Armaflex AF. Especially inside the cooling plant there are many curved pipes and the accessibility to install insulation is limited. Insufficient or improperly installed insulation will lead to the forming of ice that, when melting during warm-up periods, can cause damage to equipment in the vicinity. For a typical example see Fig. 3.

\footnotetext{
${ }^{2}$ Sub-cooled liquid is defined as a liquid at a temperature a few degrees below the boiling point at the current pressure.

${ }^{3}$ Armaflex AF is a product of Armacell ${ }^{\mathrm{TM}}$.
} 


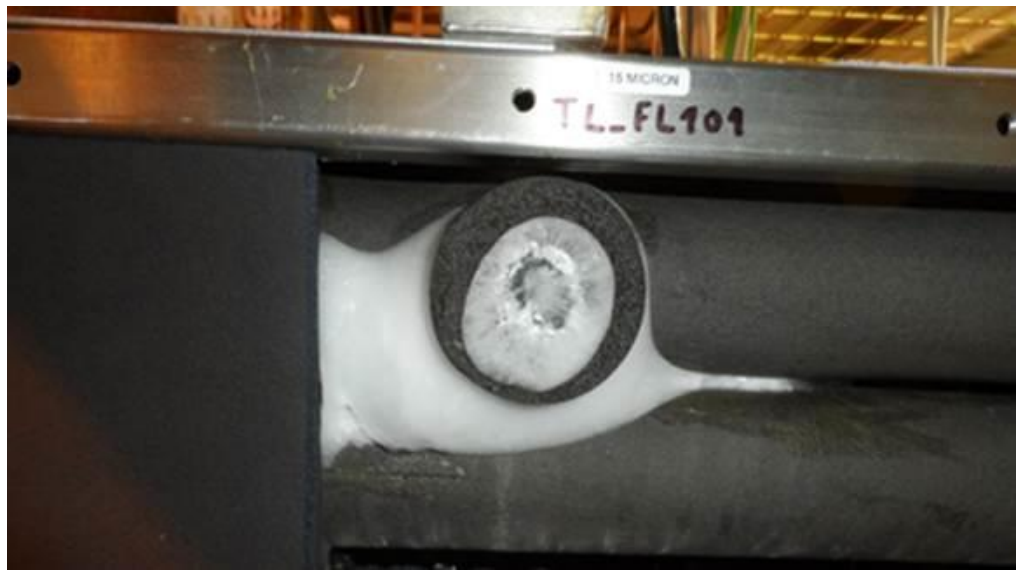

Fig. 3: Forming of ice at a spot in the cooling plant that is improperly insulated.

The cooling system is equipped with 17 electro-valves, $3 \mathrm{CO}_{2}$ pumps, 10 heat exchangers, 8 control valves, 9 heaters, 31 pressure sensors and 192 temperature sensors. The system is controlled by dedicated VELO code running in a Siemens S7-400 Programmable Logic Controller (PLC). It is optimized for minimal downtime, stable operation and highly reliable cooling performance.

The cooling plant and the PLC are placed in the radiation free zone, so they can be accessed even when there is beam in the LHC and the detector itself is inaccessible. A picture of the cooling plant is shown in Fig. 4. More details about the system can be found in [3] and [4].

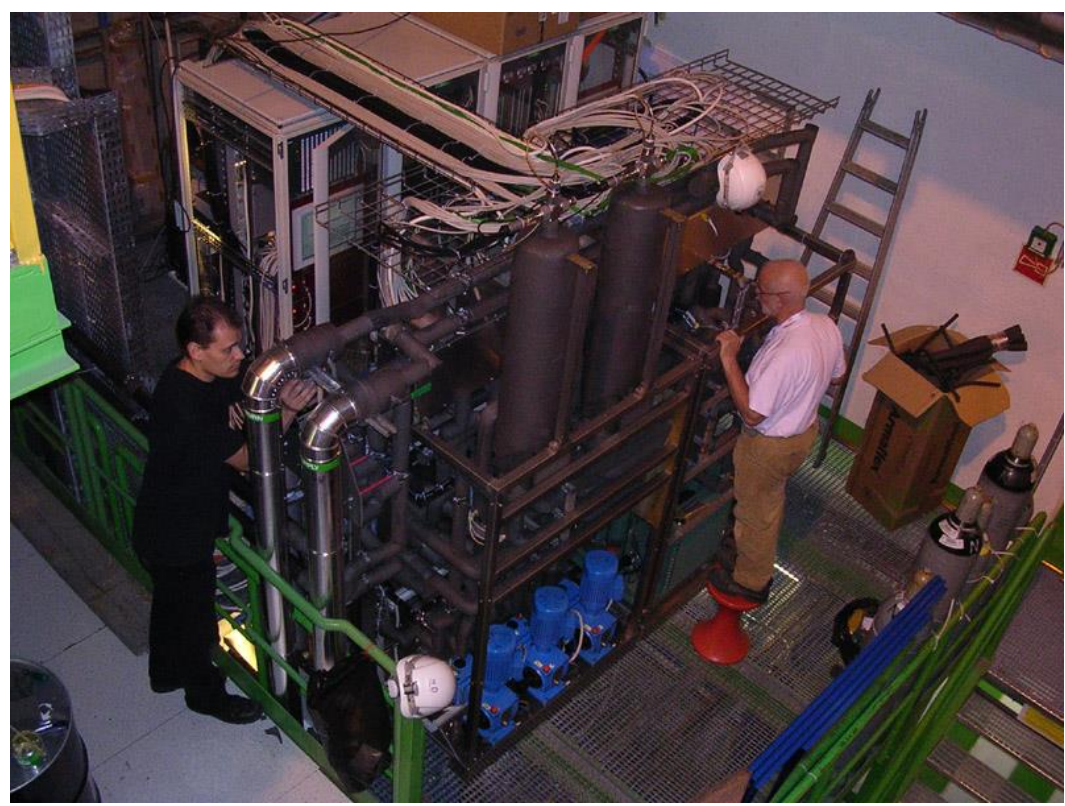

Fig. 4: Picture taken during the installation of the cooling plant in the radiation free zone. On the front left the $\mathrm{CO}_{2}$ plant with the $3 \mathrm{CO}_{2}$ pumps (blue) and the 2 accumulators (grey) on top. The freon plant with the water-cooled and air-cooled chillers is on the front right. In the back the rack with the PLC, PLCscreen and other control electronics. 


\section{Cooling performance}

The cooling performance of the system can be illustrated with the following key figures:

$\circ$ it takes one and a half hours to cool the whole system down from room temperature to the operational temperature of $-28^{\circ} \mathrm{C}$,

0 the system is flexible and easy to operate; by changing the setpoint of the accumulator the operational temperature can be varied between -30 and $-5{ }^{\circ} \mathrm{C}$,

$\circ$ after the low voltage has been switched on, i.e. the cooling system is charged with the full heat load of $800 \mathrm{~W}$, it takes 10 minutes to reach the final module temperatures and stable operation conditions of the cooling system,

o the long-term stability of the module temperatures is $<0.1{ }^{\circ} \mathrm{C}$.

During the last 4 years the cooling system performed according to its specifications and there hasn't been any need to replace a component due to malfunctioning.

\section{Filters}

To prevent clogging of components that are difficult to access or to replace, like the capillaries in the detector, eleven filters with a pore size of $15 \mu \mathrm{m}$ are installed throughout the system: one in each chiller, five in the $\mathrm{CO}_{2}$ plant and four at the VELO detector.

Over periods of months, gradual rises in the readings of certain pressure gauges of one detector half were observed. This also showed up as a slowly increasing operational temperature of that detector halve. These observations were interpreted as a possible sign of clogging filters. After renewal the contaminated filters were inspected with a Scanning Electron Microscope and much deposited debris was observed. Next, the chemical composition of the contaminations was determined with an Energy Dispersive Spectrometer, see Fig. 5.
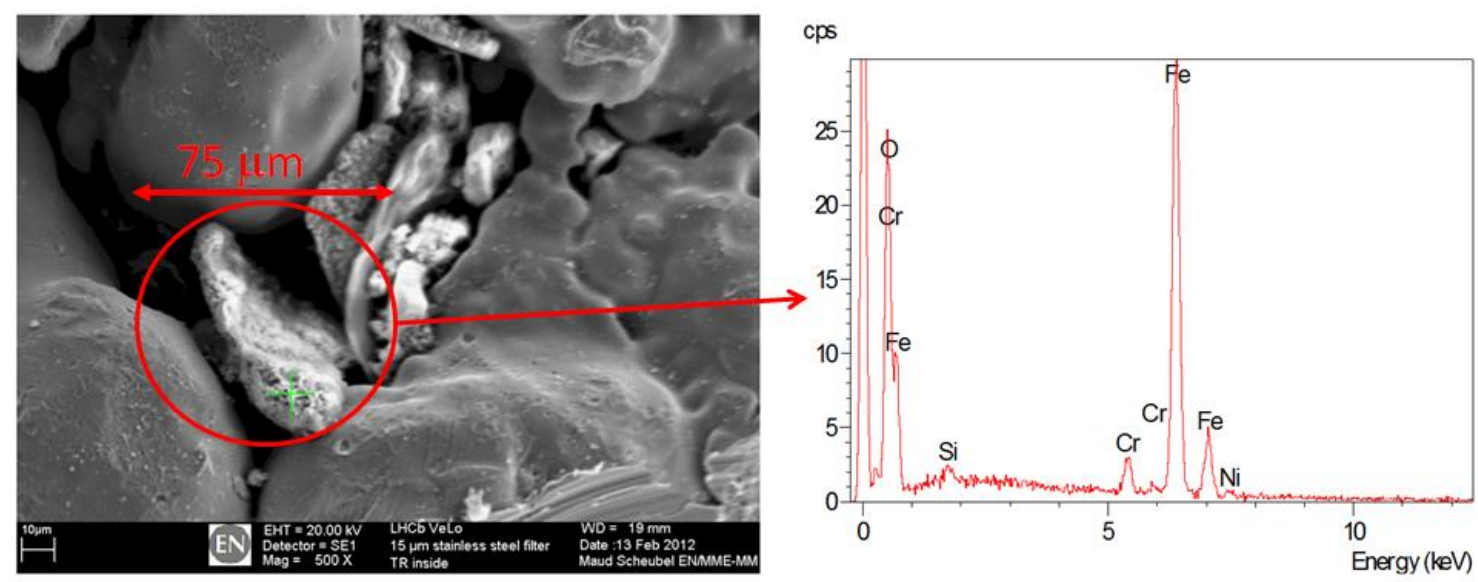

Fig. 5: Left: image of a filter taken with a scanning electron microscope. Right: spectrum obtained with an energy dispersive spectrometer of a contamination found in the filter.

Clear peaks of oxygen $(\mathrm{O})$, chromium $(\mathrm{Cr})$ and iron $(\mathrm{Fe})$ are identified, but in the past also chlorine $(\mathrm{Cl})$ has been observed. The latter might have been caused by joints that were soldered with flux for a test-beam experiment. Therefore it cannot be stressed enough how important it is to work in a clean environment and, even for small test setups, be vigilant to contamination of any part of the final system. 


\section{Superheated $\mathrm{CO}_{2}$}

After startup of the cooling system it occurs regularly that in a varying number of modules the phenomenon of super-heated ${ }^{4} \mathrm{CO}_{2}$ is observed, which evidently leads to a very poor cooling performance. Whether or not this is the case can be seen by comparing the temperatures of the modules with values elsewhere in the system, or with values recorded in the past, see Fig. 6.

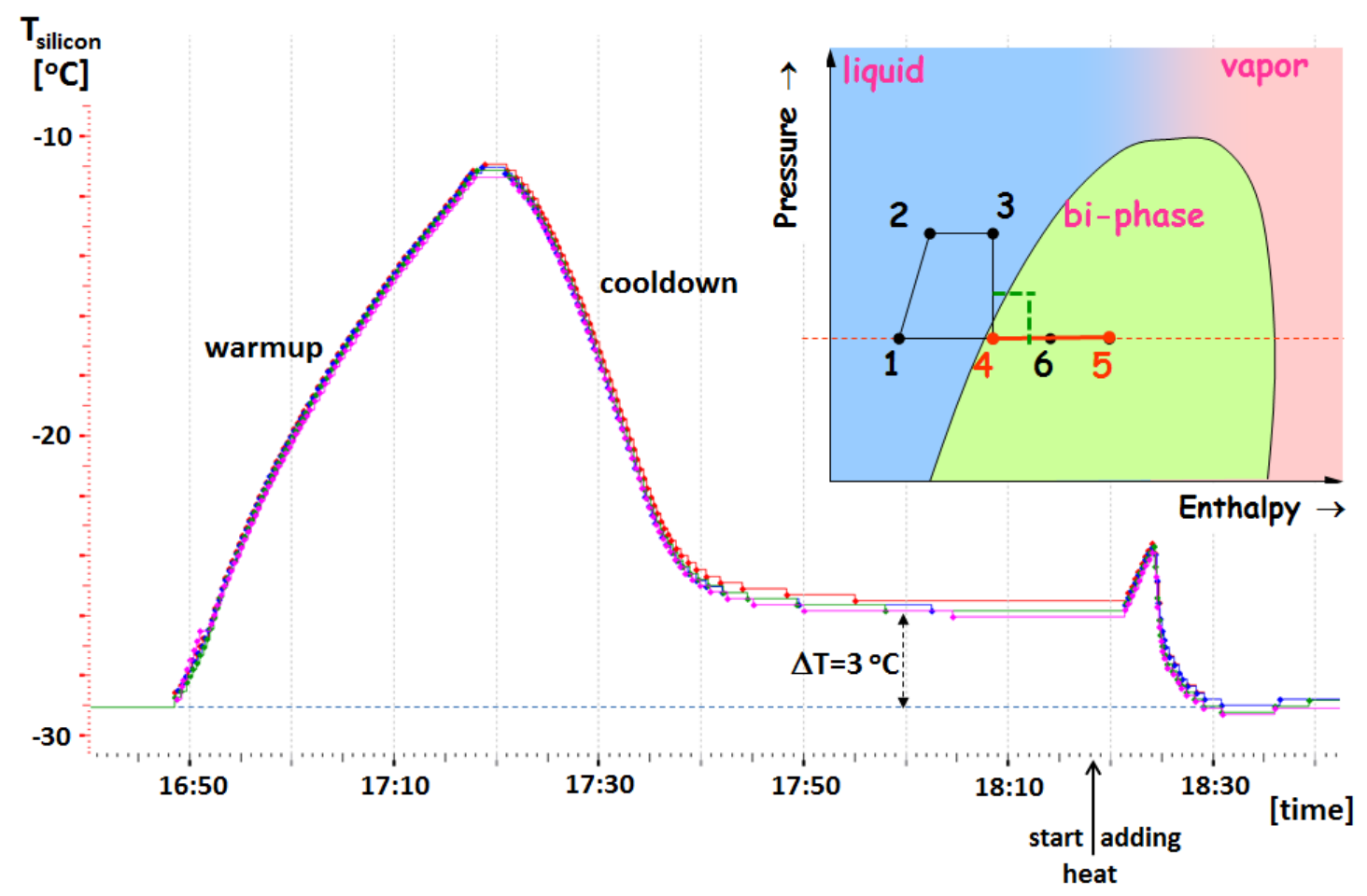

Fig. 6: Example of the effect of super-heated $\mathrm{CO}_{2}$ in four modules. After warmup and cooldown of the system the $\mathrm{CO}_{2}$ becomes super-heated, i.e. it doesn't start boiling and therefore cools very poorly. The numbers in the inset correspond with the nodes in the schematics of the cooling system in Fig. 2.

This problem is solved by increasing the enthalpy of the $\mathrm{CO}_{2}$ flow at the entrance of the detector by means of a dedicated heater. The pressure versus enthalpy diagram in the inset of Fig. 6 shows what happens: instead of the expansion bringing the $\mathrm{CO}_{2}$ from the sub-cooled liquid state (node 3) to the saturation line (node 4), the trajectory indicated with the green dashed lines is followed. Here the saturation line is first crossed in the horizontal direction until boiling has set in. Then the heater is switched off and the conditions at the entrance of the evaporator (node 4) can be described as a liquid-vapor mixture with a vapor quality ${ }^{5}$ close to zero. In the trend plot of the four temperatures in Fig. 6 this sequence is visible as a small and short temperature rise after the moment the dedicated heater is switched on at 18:19, followed by a drop of $3{ }^{\circ} \mathrm{C}$, when boiling starts.

\footnotetext{
${ }^{4}$ Super-heated liquid is defined as a liquid at a temperature a few degrees above the boiling point at the current pressure.

${ }^{5}$ Vapor quality is defined as the mass fraction of a saturated vapor-liquid mixture that is vapor, i.e. a vapor quality of zero corresponds to completely liquid. When the value reaches 1.0 all liquid has been evaporated.
} 


\section{Redundancy}

To optimize the up-time of the cooling system (see Fig. 7) a number of precautions has been taken in the design, a few of which are described below.

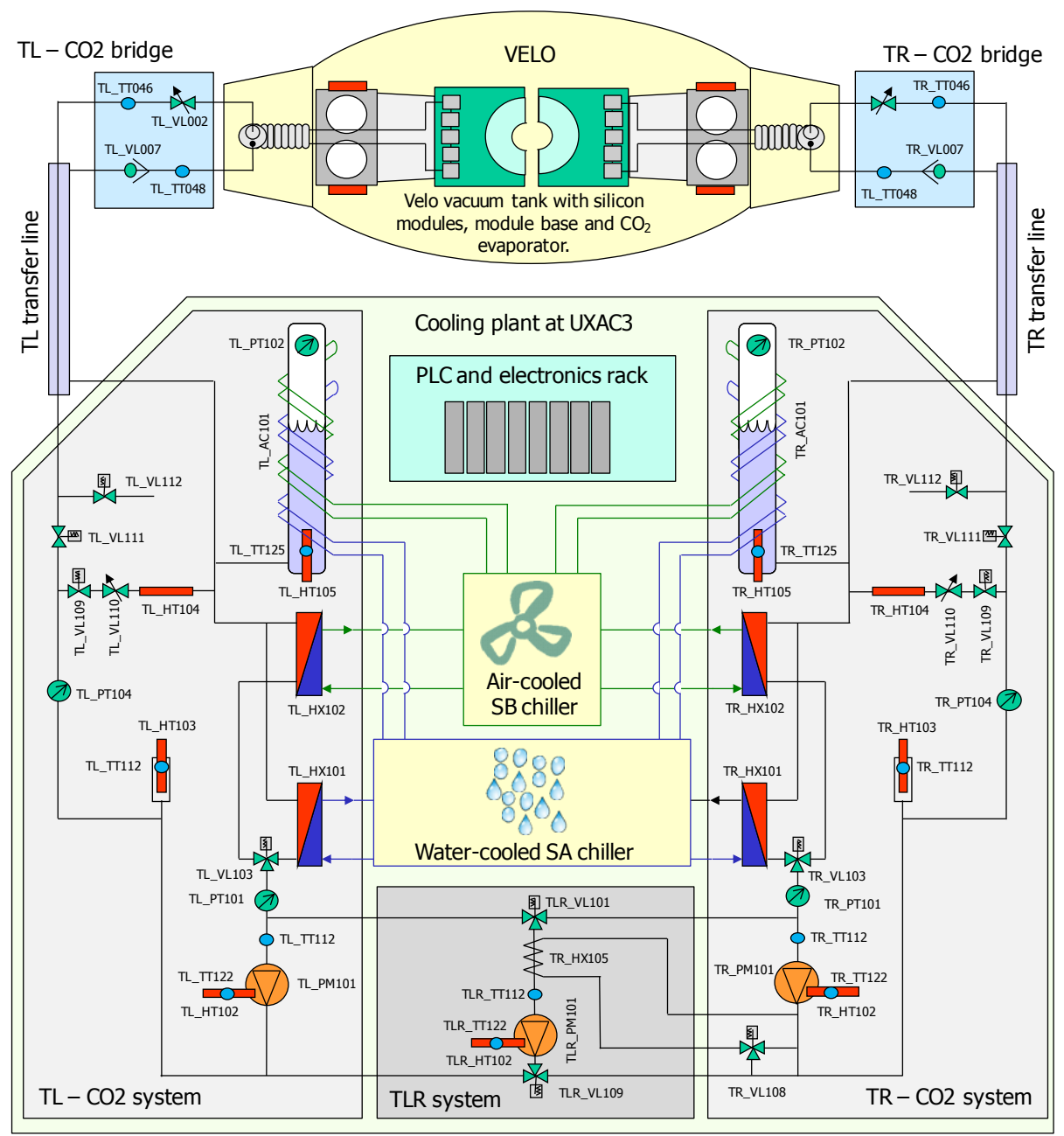

Fig. 7: Schematic layout of the VELO cooling system. From top to bottom: detector represented by two modules with 5 cooling blocks each, transfer lines, accumulators, PLC, air-cooled backup chiller, watercooled main chiller and the three $\mathrm{CO}_{2}$ pumps.

- $\mathrm{CO}_{2}$ pumps,

although only two pumps are needed, i.e. one for each detector half, three pumps are installed. The third one can take over from either of the two in case of a problem or when maintenance has to be performed,

- chillers,

the default one is a $2.5 \mathrm{~kW}$ (@-40 ${ }^{\circ} \mathrm{C}$ ) water-cooled Bitzer chiller using R507a as refrigerant. The backup is a $1 \mathrm{~kW}\left(@-25^{\circ} \mathrm{C}\right.$ ) air-cooled Bitzer chiller to keep the sensors at $-6{ }^{\circ} \mathrm{C}$, provided the front-end electronics is switched off,

- measuring devices,

all sensors that are crucial for the controls are implemented as redundant pairs, such that the system can be brought back into operation with minimal down time, in case a sensor fails. Thus far it hasn't been necessary to do so, 
- power distribution,

electrical power is supplied by the technical infrastructure of the LHCb experiment, which consists of regular single and three-phase power. For the most crucial equipment backup power is supplied by a diesel generator that becomes operational within a minute after failure of the regular power distribution. Moreover, the PLC is connected to its private Uninterruptable Power Supply (UPS) to bridge the period it takes for the diesel generator to start.

The frequency and impact of the various events causing interruptions can be summarized as follows: occasionally the chilled water supply fails. In that case the low voltage is switched off automatically and the PLC switches to the air-cooled backup chiller. In this way the sensors stay cold and it doesn't take long to bring the system back to its operational state, once the chilled water supply is back. Unfortunately, power cuts are not a rare phenomenon at CERN. Thanks to the diesel generator and the UPS of the PLC, the recovery time of the cooling system is much shorter than that of the $\mathrm{LHCb}$ experiment as a whole.

\section{Keeping the detectors cold 24/7}

The fluence that has up to now been received by the tip of the sensors is about $2 \times 10^{14} 1 \mathrm{MeV} \mathrm{n} \mathrm{n}_{\mathrm{eq}} / \mathrm{cm}^{2}$. This implies that the silicon sensors should be kept cold, i.e. below $-5{ }^{\circ} \mathrm{C}$, in order to prevent reverse annealing [5] to occur, which would increase the depletion voltage. This is quite a challenge under the present circumstances of the Long Shutdown1 (LS1) of the LHC. However, up to now the integrated warm time has been very limited: for 2012 and 2013 it amounted to about 0.5 and 0.3 days at room temperature, respectively.

\section{Warning and safety systems}

It is impossible to guarantee the continuously correct functioning of all equipment, especially during long periods with many maintenance activities on general services, while people responsible for the detector are absent. Therefore, a dedicated two-fold warning system is installed to inform people that are on call, that a certain experimental condition is not in its proper state anymore. All equipment can be controlled remotely, so no time is lost with travelling to the experimental site when a problem arises. It also significantly enlarges the community of experts that can be on call. Both systems have in common that they start sending SMSes once an experimental condition is not correct anymore.

- software based warning system,

a PVSS-subroutine ${ }^{6}$ of the experimental control system continuously checks a variety of relevant statuses, such as temperatures, pressures, valve positions, status bits and vacuum pressures. If a problem is detected it sends every hour an informative SMS to people on call, until the problem is solved.

- PLC-controlled warning system,

a GSM modem, containing a SIM card of a provider that has underground coverage at CERN, is connected via RS232 to the cooling PLC. Whenever the cooling system gets into an unwanted state the PLC starts to send SMSes to a list of phone numbers. This is repeated every half hour until the problem is solved.

The safety of the VELO detector is a key point of the design. Per detector half about $400 \mathrm{~W}$ is dissipated by the front-end electronics. Since the amount of material involved is minimal and the detector is operated in vacuum, extreme care should be taken that the detector doesn't get damaged. This would happen within a couple of seconds even in the case of a minor irregularity

\footnotetext{
${ }^{6}$ PVSS (formerly $\mathrm{ETM}^{\mathrm{TM}}$, now Siemens ${ }^{\mathrm{TM}}$ ) is a software package for process control of complex and distributed systems.
} 
in the cooling capacity. This safety requirement is fulfilled by a three-fold system, consisting of a software and a hardware branch, that act independently.

- hardware safety system,

the information of the four temperature sensors of each module is combined and serves as input for the so-called interlock box that contains a field-programmable gate array (FPGA) that can switch off the low voltage of the concerned module(s), and as such remove the heat source,

- software safety system,

132 cooling parameters are monitored continuously by a dedicated PVSS program, each of which has a warning, error and fatal limit for both the upper and lower level. For each limit violation an appropriate action is defined,

○ PLC,

the PLC can solve by itself a large number of issues by switching from the default configuration to one in which a spare component is used. For instance, in case the main chiller stops working, automatically the system switches to the backup chiller. In case one of the $\mathrm{CO}_{2}$ pumps stops working, automatically the spare pump takes over. In case the PLC cannot maintain the required cooling conditions an error flag is set, which triggers the interlock box to bring the system in a safe state.

\section{Conclusions and outlook}

The cooling system of the VELO was designed and built at Nikhef (Amsterdam) in the period 2002-2007. In 2007 it was installed in LHCb, followed by a commissioning phase that ended in 2008. It has now been operational continuously for the last four years.

The system fulfills its design specifications and fully satisfies the detector requirements. In the initial phase of the project a lot of effort was devoted to designing a reliable system that can be maintained with minimal down time. This materialized in redundancy of crucial components and implementation of the controls in a PLC. The only problems that needed to be solved over the years were clogging filters and the sub-optimal thermal insulation, which led to excessive ice-forming. Both issues are now under control. The integrated warm time during 2012 and 2013 was kept below one day, which is largely sufficient to prevent reverse annealing of the irradiated silicon sensors.

In early 2015, when LS1 has come to an end, data-taking will resume in LHCb. This Run 2 is scheduled to last till the middle of 2018, when the present VELO detector will be replaced by a pixel detector [6][7], as part of the LHCb Upgrade project [8]. The other parts of the present cooling system will then be replaced or substantially modified.

\section{References}

[1] A.A. Alves Jr et al., The LHCb detector at the LHC, JINST 3, S080005, 2008 [doi: 10.1088/1748-0221/3/08/S08005].

[2] S. Löchner et al., The Beetle reference manual, http://cdsweb.cern.ch/record/1000429

[3] B. Verlaat, Controlling a secondary 2-phase $\mathrm{CO}_{2}$ loop using a 2-phase accumulator, International Conference of Refrigeration, Beijing, ICR07-B2-1565.

[4] M.G. van Beuzekom, A. van Lysebetten, B. Verlaat, $\mathrm{CO}_{2}$ cooling experience ( $\left.\mathrm{LHCb}\right)$, in proceedings of The $16^{\text {th }}$ International Workshop on Vertex detectors, Lake Placid, NY, USA, PoS(Vertex 2007) 009. 
[5] M. Moll, Radiation damage in silicon particle detectors - microscopic defects and macroscopic properties, DESY-THESIS-1999-040.

[6] M.G. van Beuzekom, The LHCb Vertex Locator (VELO) Upgrade, These Proceedings, PoS(Vertex2013)016.

[7] The LHCb collaboration, LHCb VELO Upgrade Technical Design report, CERN-LHCC-2013021, LHCB-TDR-013, 2013.

[8] The LHCb collaboration, LHCb Upgrade Letter of Intent, CERN-LHCC-2011-001, 2011. 